\title{
Cardiac Cephalgia: Angina in the Head
}

\author{
*Rahman $\mathrm{MM}^{1}$, Razzaque $\mathrm{MA}^{2}$, Alam I ${ }^{3}$, Iqbal A ${ }^{4}$, Mallick GR ${ }^{5}$, Munshi $S^{6}$,Wareshuzzaman $\mathrm{M}^{7}$, E-Hasan $\mathrm{AKMQ}^{8}$
}

\begin{abstract}
Cardiac cephalgia is a migraine like headache that occurs during episodes of myocardial ischaemia. Although most of the patients presenting with ischaemic heart disease have chest pain, there are other rare presenting symptoms like cardiac cephalgia. Headache can be the only presentation of coronary artery disease. We report a case of a 57 years-old man, Presenting with only headache during brisk walking, Exercise Tolerance Test (ETT) was positive for Electrocardiograph (ECG) evidence of provocable myocardial ischemia, who latter was diagnosed as double vessel coronary artery diseaseon Coronary Angiogram (CAG). As the patient preferred remaining without revascularization, he was put onto optimum medical management for ischaemic heart disease. A follow up visit after one month revealed, marked improvement of the headache with anti anginal medications. Early evaluation and diagnosis of the headache symptom should be
\end{abstract}

1. *Professor Dr. Md. Mahbubu Rahman, Project Director, Establishment of 500-Beded Hospital \& Ancillary Buildings in Jessore, Cox's Bazar, Pabna and Abdul Malek Ukil Medical College (AMUMC) and Jononeta Nurul Hoque Adhunik Hospital, Noakhali Project, DGHS, MOHFW, Bangladesh. Former Professor of Cardiology, National Institute of Cardiovascular Diseases, Dhaka. Mobile: +8801714103922, Email: mahbubbabu25@gmail.com.

2. Dr. Md. Aminur Razzaque, Assistant Professor, Department of Cardiology, National Institute of Cardiovascular Diseases, Dhaka.

3. Dr. Iftikher Alam, Assistant Professor, Department of Neurology, Dhaka Medical College Dhaka.

4. Dr. Asif Iqbal, Curator, Institute of Epidemiology, Disease Control and Research, Dhaka.

5. Dr. Golam Rahman Mallick, Assistant Professor, National Institute Of Cardiovascular Diseases.

6. Dr. Swati Munshi, Specialist, Department of Radiology, Square Hospital.

7. Dr. Md. Wareshuzzaman, Assistant Professor, Department of Cardiology, National Institute of Cardiovascular Diseases, Dhaka.

8. Dr. A. K. Mohammad Qudrath-E-Hasan, Medical Officer, Department of Cardiology, National Institute of Cardiovascular Diseases, Dhaka.

*For Correspondance done because treatment with anti-migraine drugs may deteriorate headache and undermine the diagnosis of coronary artery disease.

Keywords: Cardiac cephelgia, coronary artery disease, headache.

\section{INTRODUCTION}

Headache is found in $6 \%$ of all cardiac ischaemic cases. ${ }^{1}$ According to the International Classification of Headache Disorders (ICHD) $3^{\text {rd }}$ edition cardiac cephalgia is a 'migraine like' headache, usually but not always aggravated by exercise, occurring duringepisode of myocardial ischaemia and it is relieved by nitroglycerine. ${ }^{2}$ Cardiac cephalgia is a typical secondary headache disorder, usually initiated by exertion that is related to myocardial ischaemia. Primary exertional headachesuch as sex cough or exercise induced headache are typically benign. Cardiac cephalgia, on the other hand, can have life threatening complications thereare a total of 36 cases that have been reported in literature so far. ${ }^{3}$ We report a case of a 57 year old male, presenting to a neurologist with headache as sole symptom, who later was diagnosed having severe coronary artery disease.

\section{CASE REPORT}

A 57 year old Muslim male, hypertensive, diabetic, nonsmoker without any positive family history of ischaemic heart disease, presented to us with a history of post exertional headache for last 2 years. It spread to the occipital region and back of neck after exertion. Initially it occurred in moderate to severe exertion, later worsened and presently it occurs with minimal exertion relieves on taking rest. The headache was not associated with watering of eyes, nausea or vomiting. With this complaint he presented to a neurologist and underwent neurological examinations and investigations including magnetic resonance imaging (MRI) of brain and cervical spine, Magnetic resonance venogram (MRV) and carotid duplex study which failed to reveal any abnormality.

Initially diagnosed as a case of migraine and treated accordingly without any improvement, the patient underwent an exercise tolerance test (ETT). During the test, he developed headache without chest pain. It was associated with ST-T change in stage-1 which persisted up to recovery stage. Accordingly sublingual nitrate was given which relieved headache. 


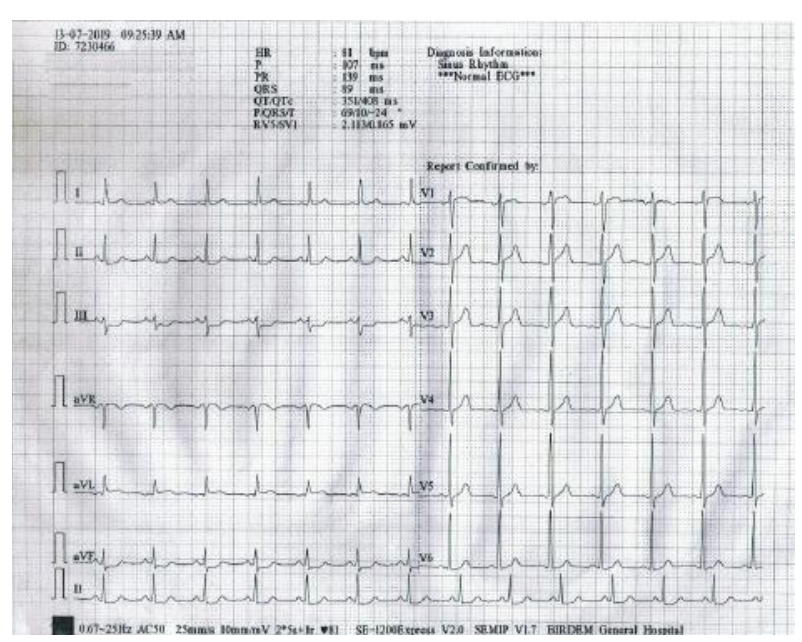

Fig.-1: A) Resting ECG showing mild ST depression at inferior leads

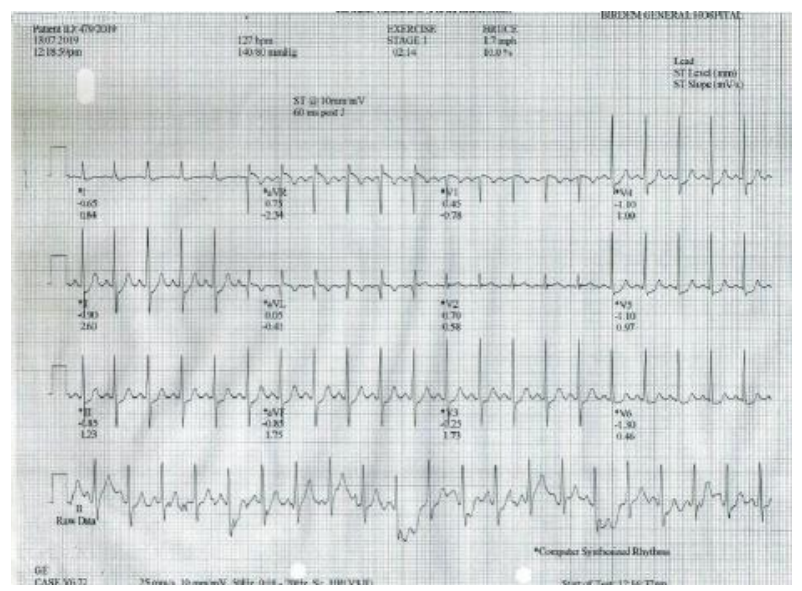

Fig.-1: B) ST change at inferior and lateral leads in stage 1 of ETT

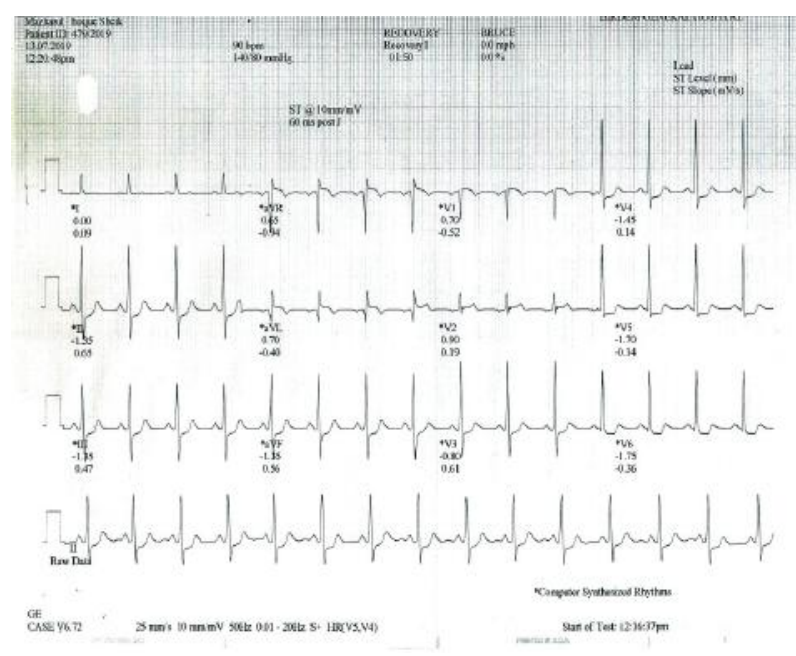

Fig.-1: C) ST change at inferior and lateral leads in recovery stage of ETT
Few days later, coronary angiogram was done which revealed 99\% stenosis in proximal Left circumflex coronary artery (LCX), total occlusion of proximal Right coronary artery (RCA) and minor plaques in left anterior descending (LAD) artery at proximal part. Carotid and vertebral arteries were free of disease.

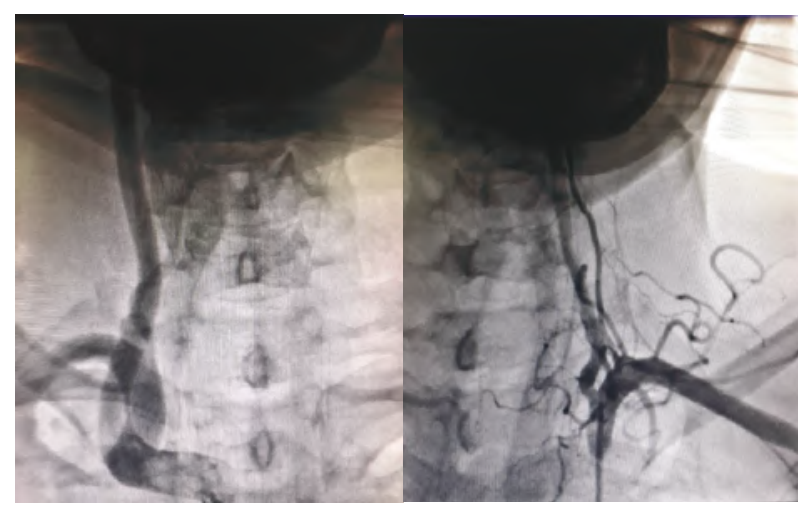

Fig.-2: Angiographic view of Right and left carotid and vertebral artery showing no stenosis.

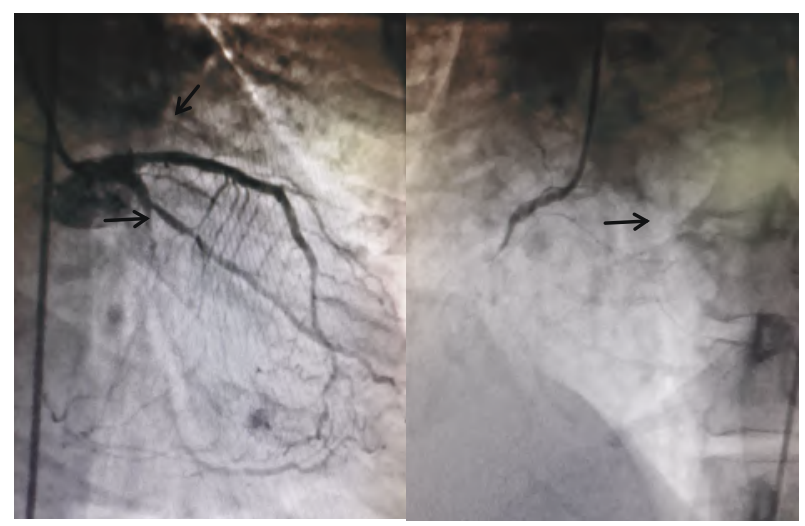

Fig.-3: Coronary angiogram showing diseased coronary vessels.

Patient was advised revascularization with angioplasty. But due to some constraints patient refused to do this and we discharged him with optimum medical management. On follow up visit after one month symptomatic improvement was found with anti anginal drugs.

\section{DIFFERENTIAL DIAGNOSIS}

It is important to distinguish cardiac cephalgia from migraine with or without autonomic symptoms and other forms of exertional headache.

\section{DISCUSSION}

The term Cardiac Cephalgia was coined by Lipton et al. ${ }^{4}$ In 1997 as a form of exertional headache. The diagnosis of 
Cardiac cephalgia depends on the presence of severe headache worsened by physical exercise or stress and relieved with rest and or administration of nitrates. The International Headache Society has included Cardiac cephalgia as a specific entity in its international classification of Headache Disorders and proposed diagnostic criteria. ${ }^{7}$

A. Headache, which may be severe, aggravated by exertion and accompanied by nausea and fulfilling criteria "C" and "D".

B. Acute Myocardial ischaemia.

C. Headache develops concomitantly with acute myocardial ischaemia.

D. Headache resolves and does not recur after effective medical therapy for acute myocardial ischaemia or coronary revascularization.

Our case meets each of the mentioned criteria. Cardiac Cephalgia is also called by several synonyms such as angina capitis, angina cranialis or simply as 'headache angina., 6

Bini et $\mathrm{al}^{5}$ reviewed 30 cases with cardiac cephalgia and showed a mean age of 62.4 years (range 35-85 years). Pain is usually not localized, and may be unilateral or bilateral. Pain is almost always severe and has been described as having different characteristics. There may or may not be other accompanying symptoms and if there are, 30\% may have autonomic in nature. In $27 \%$ of the cases headache is the only manifestation of a cardiovascular ischaemia. The headache starts immediately after physical exertion. In $33 \%$ cases headache appeared at rest. The frequency of this headache is highly variable and is $57 \%$ of patients show ECG abnormalities at rest $^{7}$ as well as elevated cardiac enzymes $^{8}$ and in the remaining ECG changes appear only during stress. ${ }^{9}$

Four theories have been proposed as a pathophysiologic mechanism. The first suggests that Cardiac cephalgia is a referred pain, as there is a connection between central nervous system (vagus nerve) and the cranial pain afferents (trigeminal nerve) is the upper part of the spinal cord. ${ }^{4,10}$ The second theory proposed that cardiac cephalgia is secondary to elevated intracranial pressure due to venous stasis caused by transient decrease in cardiac output due to ischemic ventricular dysfunction. ${ }^{4}$ According to the third theory, it is secondary to the local release in the heart muscle of the chemical mediators capable of inducing remote pain, in this case headache. ${ }^{4}$ Among others, serotonin, bradykinin, histamine and substance $\mathrm{P}$ have been proposed as potential pain producing substances. The increase in intra-cardiac pressure during anginaattacks could also result in release of natriuretic peptides with consequent vasodilatation of cerebral vasculature resulting in headache.

Finally Cardiac cephalgia could be due to concomitant presence of vasospasm in both coronary and cerebral vasculature. ${ }^{11}$

When the headache occurs as the only manifestation of as acute coronary event, the diagnosis could be difficult if occurred in our case. Useful clues are older age at onset, no previous history of headaches, and presence of risk factors for cardiovascular disease and the onset of headache under stress. Misdiagnosis as migraine and prescribing anti-migraine drug may cause suffering of the patient. Even delayed diagnosis can be fatal. So awareness should be build up among the concerned physicians and further study is needed to see the prevalence of headache in IHD patients.

\section{CONCLUSIONS}

Headache is a common symptom associated with various clinical conditions. It is rarely considered in cardiac diseases. Cardiac cephalgia though a very rare condition should not be underestimated. As if not properly addressed it may culminate into serious conditions like acute coronary syndrome and may even be life threatening. When the headache occurs as the only manifestation of an acute coronary event, the clues for suspicion are a) older age at onset, b) no past medical history of headache, c) presence of risk factors for vascular disorders and $\mathrm{d}$ ) onset of headache under stress ${ }^{5}$.

\section{REFERENCES}

1. Wei J.H. Wang H.F. Cardiac cephalgia case report and review. Cephalgia International Journal of Headache 2008; 288:892-6

2. Headache Classification Committee of the International Headache Society (IHS). The International Classification of Headache Disorder, 3rd edition (beta version) Cephalgia 2013; 33:629-808

3. Wasse I. N. Ali A. T. Kalsanevaki A. Z.Cardiac Cephalgia.Cardiol Res 2014; 5(6):195-197

4. Lipton RB, Lowenkopf T, Bajwa ZH, Leckie RS, Ribeiro S, Newman LC, Greenberg MA. Cardiac 
cephalgia: a treatable form of exertional headache. Neurology. 1997;49(3):813-816. doi: 10.1212/ WNL. 49.3.813.

5. Bini A, Evangelista A, Castellini P, Lambru G, Ferrante T, Manzoni GC, Torelli P. Cardiac cephalgia. The Journal of Headache and Pain. 2009;10:39. doi: 10.1007/s10194-008-0087-x.

6. Sathirapanya P (2004) Anginal cephalgia: a serious form of exertional headache. Cephalalgia 24(3):231-234

7. Seow VK, Chong CF, Wang TL, Ong JR. Severe explosive headache: a sole presentation of acute myocardial infarction in a young man. Am J Emerg Med. 2007;25(2):250-251. doi: 10.1016/j.ajem. 2006.11.014.
8. Korantzopoulos P, Karanikis P, Pappa E, Dimitroula V, Kountouris E, Siogas K (2005) Acute non-St-elevation myocardial infarction presented as occipital headache with impaired level of consciousness. Angiology 56:627-630

9. Lanza GA, Sciahbasi A, Sestito A, Maseri A. Angina pectoris: a headache. Lancet. 2000;356(9234):998. doi: 10.1016/S0140-6736(00)02718-5.

10. Meller ST, Gebhart GF. A critical review of the afferent pathways and the potential chemical mediators involved in cardiac pain. Neuroscience. 1992;48(3):501-524. doi: 10.1016/0306-4522 (92)90398-L.

11. Ramadan NM. Headache caused by raised intracranial pressure and intracranial hypotension. CurrOpin Neurol. 1996;9(3):214-218. doi: 10.1097/00019052 -199606000-00011. 